\title{
RINGO3: a multi-colour fast response polarimeter
}

\author{
D. M. Arnold, I. A. Steele, S. D. Bates, C. J. Mottram, R. J. Smith \\ Astrophysics Research Institute, Liverpool JMU, Twelve Quays House, Egerton Wharf, \\ Birkenhead CH41 1LD, UK
}

\begin{abstract}
GRB jets contain rapidly moving electrons which will spiral around magnetic field lines. This causes them to emit polarized synchrotron emission. We have built a series of polarimeters (RINGO and RINGO2) to investigate this by measuring the polarization of optical light from GRBs at a certain single wavelength. The instruments are mounted on the Liverpool Telescope, which is a fully robotic (i.e. unmanned) telescope on La Palma which reacts to triggers from satellites such as the NASA SWIFT mission. This has had great success, with the first ever detections of early time optical polarization being made. In addition, the first measurements of the change in optical polarization from a GRB as the jet expands have recently been obtained.

In this paper we describe the design and construction of RINGO3. This will be a multi-colour instrument that can observe simultaneously at three wavelengths. By doing so we will be able to unambiguously identify where in the burst the polarized emission is coming from. This will allow us to distinguish between three possibilities: (1) Magnetic instabilities generated in the shock front, (2) Line of sight effects and (3) Large-scale magnetic fields present throughout the relativistic outflow. The instrument design combines a rapidly rotating polaroid, specially designed polarization insensitive dichroic mirrors and three electron multiplying CCD cameras to provide simultaneous wavelength coverage with a time resolution of 1 second.
\end{abstract}

Keywords: robotic telescopes, gamma ray bursts, polarimeters, astronomical instrumentation

\section{INTRODUCTION}

The Liverpool Telescope (LT) ${ }^{1}$ is a fully robotic 2.0 metre class telescope at Roque de los Muchachos Observatory on La Palma. Operated by Liverpool John Moores University, it has been at the forefront of time variable and rapid reaction astronomy since 2004. A principal science goal of the LT has been in the area of rapid follow up ( $<3$ minutes) of the optical transients associated with Gamma Ray Bursts (GRBs). The Telescope has been optimised for this role with fast slewing, an innovative clamshell dome and quick selection between instruments on the Acquisition \& Guidance (A\&G) unit which can host 5 instruments. Since 2006, the autonomous LTTRAP pipeline ${ }^{2}$ has been responding to GRB triggers from the SWIFT satellite and producing automated early-time multicolour lightcurves of GRB afterglows. The pipeline will often detect (if visible) the transient in the field, with a high confidence, and select the best course of observations based on object magnitude (i.e. further photometry, polarimetry or even spectroscopy for the brightest bursts). The success of this autonomous operation has enabled the photometric early-time evolution of GRBs to be observed, starting from 1 minute post trigger.

Theoretical models of GRB emission are focused on the expanding fireball model, whereby shells in a jet of relativistically expanding plasma collide. This transfers a huge amount of kinetic energy to the electrons which spiral around strong magnetic fields, producing synchrotron emission that can be highly polarised (up to 70\%) in the case of large scale ordered fields. When the jet interacts with the circumburst medium, it is predicted to create a reverse shock, which propogates back through the jet. We have developed two different mono-band polarimeters for the Liverpool Telescope which have made ground-breaking discoveries in this field. RINGO ${ }^{3}$ was successful in the first ever detection of polarisation within a GRB afterglow (GRB 090202) and RINGO2 ${ }^{4}$ has been successful in the detection of the first ever time varying polarisation (GRB 101112). In order to distinguish the area of the jet in which the polarised signal occurs we need to perform photometry in different bands. It is possible to identify certain times and wavelengths which are dominated by emission from a reverse shock. By performing multi-band polarimetry we will be able disentangle the radiation from different emitting regions and probe the magnetic structure of the interior of the jet.

Further author information: (email dma@astro.livjm.ac.uk) 


\section{SCIENCE CASE}

Figure 1 shows three representations of a GRB outflow in the context of the standard fireball model for a variety of magnetic field structures and different orientations to the observer's line of sight (optical axis). A large degree of polarization is predicted when the ejected material is threaded with a large-scale ordered magnetic field as shown in (a) and is the favoured model to explain the measured polarization in GRB 090102 of $>10 \%$. Alternatively, if no ordered magnetic field is present and, instead a tangled magnetic field is produced in the shock front, the detected light will be polarized only if the observer's line of sight is close to the jet edge (b). A compromise is shown in (c) in which the shock front contains a number of independent patches of locally-ordered magnetic fields, which may explain the evolution of polarization in the forward shock dominated GRB 101112. In order to distinguish between these models we need high time resolution, multi-band polarimetry. By extending the design of RINGO2 (see Section 3) to be capable of simultaneous multi-band imaging, we will be able to distinguish between these three models on a burst by burst basis.
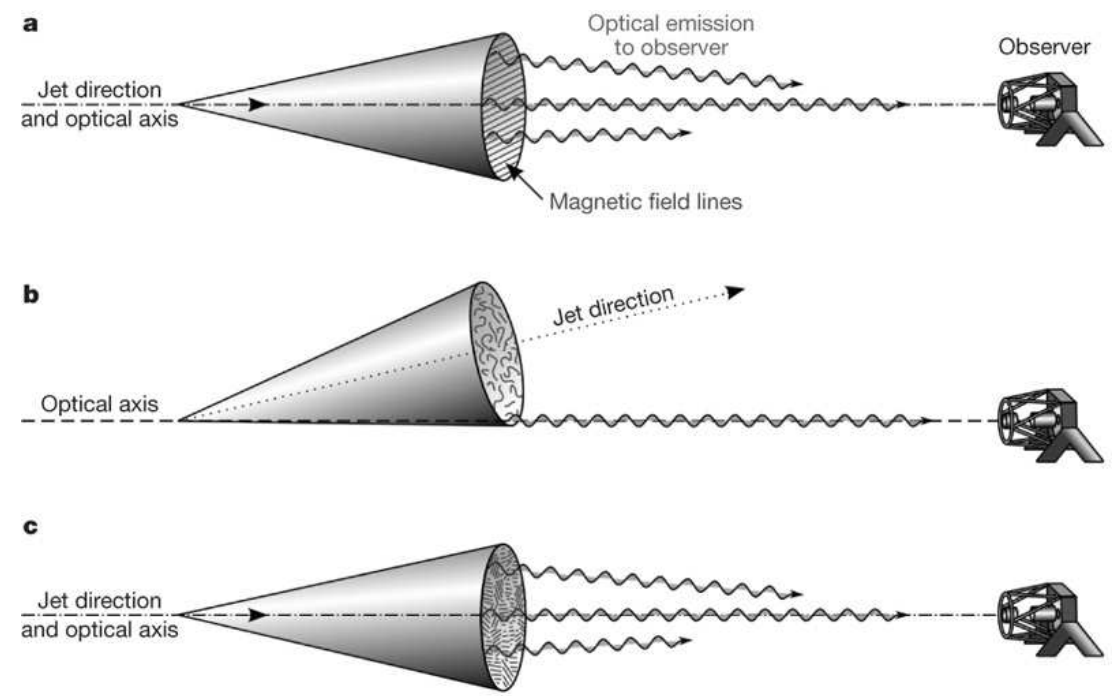

Figure 1. Competing models of GRB magnetic field structure.

\section{RINGO AND RINGO2}

GRBs are transient phenomena, with the optical afterglow fading rapidly within the first hour of observation. Traditional methods of polarimetry (taking two consectutive exposures through two orientations of a polarising filter) are unsuitable for variable sources (such as GRBs) where the brightness can change significantly on the timescales of the observation. To create a polarimeter suitable for the measurment of GRB optical afterglows, the original RINGO instrument was built using a novel design developed by Clarke and Neumayer, ${ }^{5}$ which was unaffected by fluctuations of source brightness during the integration time. To do this it employed a rapidly rotating polaroid $(\sim 500 \mathrm{rpm})$ attached to a refracting wedge to create ringed images of each object in the 4.6 x 4.6 arcmin field*. By splitting the ring into 8 intensity bins, the Stokes parameters for polarisation could be calculated. This design had flaws however, namely the spead of the flux on the CCD into an annulus extended the area of sky background, and thus limited accurate polarimetry to only the brightest of GRBs (R 15 or brighter). Only two were observed in RINGO's four years of operation, consistent with expected rates. The other main issue was that RINGO's constant integration (typically 60secs) provided an average value of polarisation over the integration time, and was insensitive to variation in polarisation magnitude or angle fluctuations over this time.

\footnotetext{
${ }^{*}$ This size of field relates to the positional error of the Swift Burst Alert Telescope (BAT) trigger, so that $90 \%$ of GRBs
} will be positioned in the field. 
To address these two issues, we developed a completely new polarimeter design, exploiting the fast readout and low noise characteristics of electron multiplying CCD (EMCCD) cameras. This design dispenses with the refracting wedge and ringed star images, instead producing standard images. The EMCCD camera is triggered externally by the rotation of the polaroid, 8 times per rotation. Performing photometry on the 8 frames is analogous to measuring the flux on the 8 ring segments of RINGO and the same mathematics from reference ${ }^{5}$ is used to perform polarimetry calculations. By having standard images, the sky background noise is reduced, which enables accurate polarimetry of fainter sources and any number of frames of the same polaroid orientation can be stacked for polarimetry, providing a time resolution limited only by the source brightness. RINGO2 ${ }^{4}$ was built using this new design. An ANDOR Xion+ 897 camera was used, which incorporated an E2V CCD97 chip (512 x $51216 \mu \mathrm{m}$ pixels) and Peltier cooling with external triggering options allowing up to 35 frames per second. The small size of the chip allows the fast readout rates, and setting a field of view of $4 \times 4$ arcminutes gives a pixel scale of 0.4 arcsec. The ANDOR CCD is triggered by a proprietary circuit which takes the signals from 8 proximity sensors positioned around the polaroid, which are triggered in turn by the polaroid rotation. The circuit incorporates the shutter signal from the camera (indicating the camera is ready) and also a further 'home' proximity sensor, which defines the start of the polaroid rotation. The camera will only be triggered once ready, and the home position has been passed. The polaroid is rotated at $60 \mathrm{rpm}$, providing 8 frames per second. RINGO2 is a single band instrument using a wide, custom filter which approximates to the range of the Bessell ${ }^{6} \mathrm{~V}$ and $\mathrm{R}$ bands. This new design extended the range of observable GRBs for polarimetry from a limiting magnitude of $\mathrm{R} \sim 15$ to $\mathrm{R} \sim 17$, providing an expected rate of observable GRBs to be of the order of 10 in a 4 year period.

\section{DESIGN}

In order to achieve a true simultaneous multi-band instrument, it is necessary to split the incoming beam into 3 wavebands directed to each of the cameras. This splitting occurs in the collimated beam of the instrument, using dichroic mirrors which will transmit or reflect radiation based on its wavelength relative to a defined cutoff. Dichroic mirrors typically have excellent transmission and reflective efficiencies (up to 95\%), but are not polarisation insensitive and have varying efficiencies based on the polarisation angle of incident radiation, hence changing the polarisation of the beam. This issue has been addressed during the construction of MPEs GROND 7-channel imager, ${ }^{7}$ which has been developed for accurate simultaneous photometry of GRB afterglows. The specially manufactured dichroics for the GROND instrument have reduced polarisation sensitivity and we have obtained details for these. In operation, the small amount of instrumental polarisation from the dichroics will be characterised for each camera using unpolarised standard stars. The instrumental polarisation of RINGO2 is mainly due to the science fold mirror which directs the telescope beam to one of the side ports on the A\&G box and is well understood.

Two ANDOR Xion+ 897 EMCCD Cameras have been obtained, the third will be re-used from RINGO2 along with the instrument mounting and polaroid rotation mechanism. We opted to use two different models of the camera, which have different CCD quantum efficiency (QE) profiles. The BV camera is identitical to the one on RINGO2 and the BB camera is optimised for the blue end of the operating wavelength range (see Figure 2) and will be used for our lowest wavelength band. The field of view will remain at the RINGO2 standard of $4 \times 4$ arcmins and hence we will be using the same medium format Mamiya $150 \mathrm{~mm} \mathrm{f} / 3.5$ collimator lens and three $35 \mathrm{~mm}$ format Nikon AF-D 50mm f/1.4 camera lenses. Figure 3 shows the construction of RINGO3 and the main optical elements. RINGO3 extends the RINGO2 operating wavelength range of $\sim 460-740 \mathrm{~nm}$ to $\sim 400-900 \mathrm{~nm}$. In order to maximise the amount of flux detected, we have chosen not to use any standard filters for each of the 3 wavebands. Instead, the dichroic mirrors will define the band boundaries, with the falling quantum efficiency of the detectors defining the high and low wavelength band limits. This extended wavelength range means we have had to use a different polarising filter to RINGO2 in order to have a good throughput over the full range. We have chosen a Versalite precision photo-etched wire grid polaroid supplied by Meadowlark Optics. This wire grid polariser design also improves the contrast ratio of our polaroid, (the ratio of flux in / out of two polarising filters orientated at $90^{\circ}$ to each other) which will lead to more accurate photometry. We also performed extensive throughput testing on the lenses (as detailed in Section 5.1) which was needed for full characterisation for modelling purposes. Being DSLR photographic lenses, there is no guarantee of their coating 
performance in the higher near-IR wavelengths $(>760 \mathrm{~nm})$. However our testing found them to still be suitable for RINGO3.

Mechanically, the instrument design has undergone finite element analysis (FEA) for all the orientations on the sky. Casing design and camera mountings points have been iterated to reduce weight and provide the minimum amount of flexure. The areas of highest deformation are well within tolerances with deflection of less than $2 \mu \mathrm{m}$.

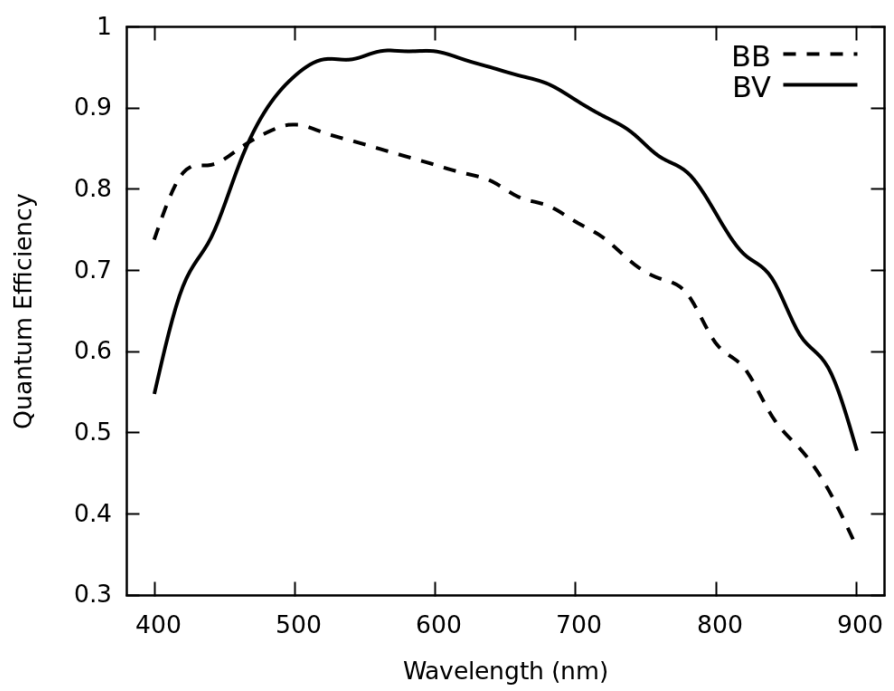

Figure 2. Plot of Quantum Efficiency of the two different models of Andor Xion CCD camera ordered for RINGO3. The $\mathrm{BB}$ model was chosen to maximise flux obtained in the lowest of our 3 wavelength bands.

\section{WAVEBAND DETERMINATION}

The main design requirement for the 3 wavebands, is that a 'typical' GRB afterglow would provide equal detected flux in each band. In order to do this we needed to create a model of instrumental efficiency and then input the spectra of GRBs. The sky spectra was also obtained for La Palma ${ }^{8}$ and put through our instrument. With the negligible dark noise and readout noise of EMCCD cameras the sky noise is dominant and hence was the only source of noise we modelled.

\subsection{Lens Throughput Testing}

Throughput testing was undertaken for all of the lens elements of RINGO3, using the two models of Andor Xion CCD cameras with a wavelength range of $400 \mathrm{~nm}$ to $900 \mathrm{~nm}$. This was done by focusing a monochromator slit onto the CCD camera, using both the camera and collimator lenses. In order to cover the full wavelength range, two different bulbs were used with the monochromator. The initial $24 \mathrm{~V}$ incandescent bulb provided a good illumination for wavelengths from 500nm upwards, but provided little flux in the 400nm and lower range of wavelengths, where a $5 \mathrm{~V}$ Blue LED with a known output spectrum was used. The monochromator itself has a throughput and the detecting EMCCD camera has a certain quantum efficiency of photon detection, both dependent on wavelength. In order to account for these a separate baseline set of data was obtained by having the monochromator slit pushed right up against the Andor CCD chip window creating an unfocused baseline. For all these tests the Andor CCD camera had a gain setting of 1 and the chip was cooled to an operational temperature of $-60 \mathrm{C}$ reducing thermally induced dark noise to negligible levels. Exposure times were chosen to only saturate the CCD pixel well depth up to 75\% (relating to about 9000 counts).

The lens data was reduced by creating a rectangular image region that contained the full slit image. A second identical background region was also defined close to the slit but containing no slit flux. The counts induced in our detector from the monochromator were then calculated by subtracting the total counts in the background 


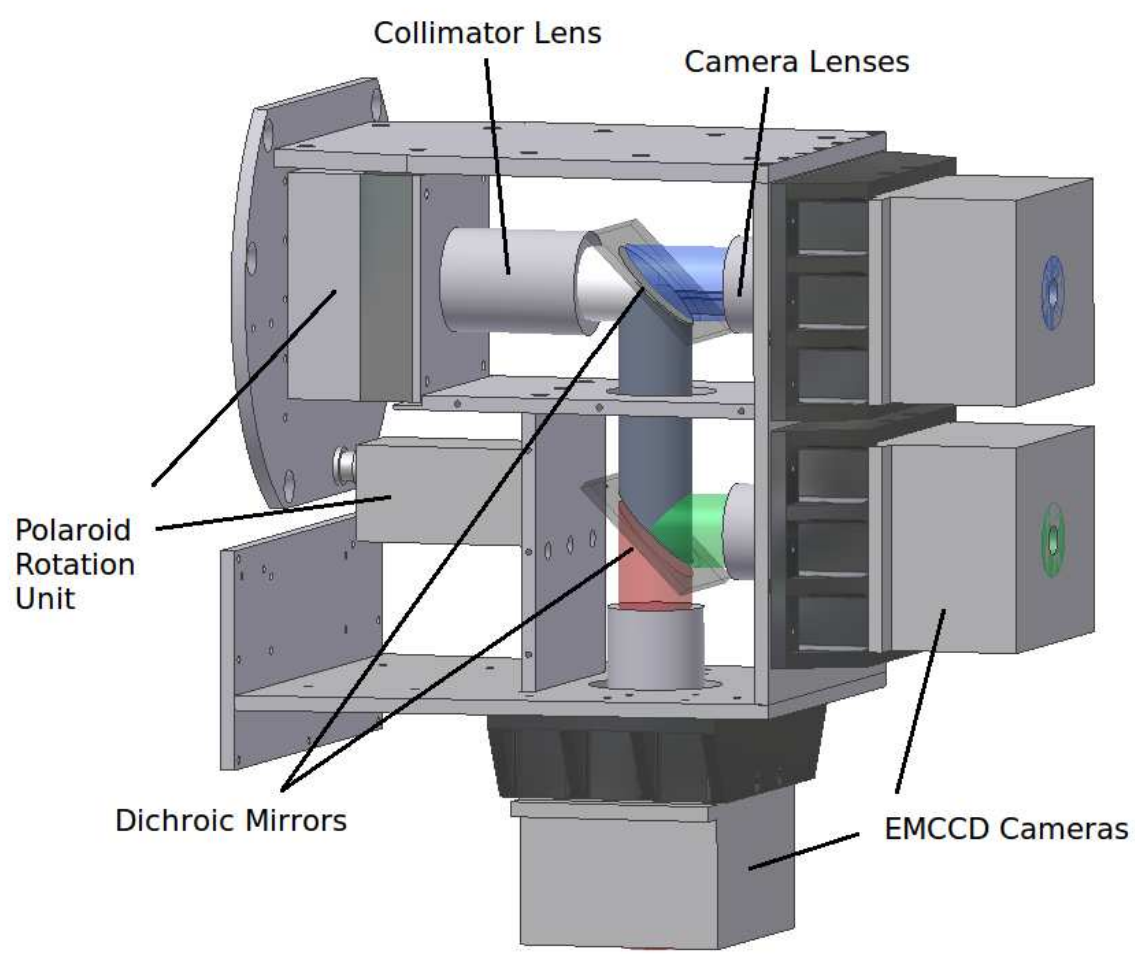

Figure 3. The mechanical design of RINGO3, with sides removed, showing the optical layout and beam paths.

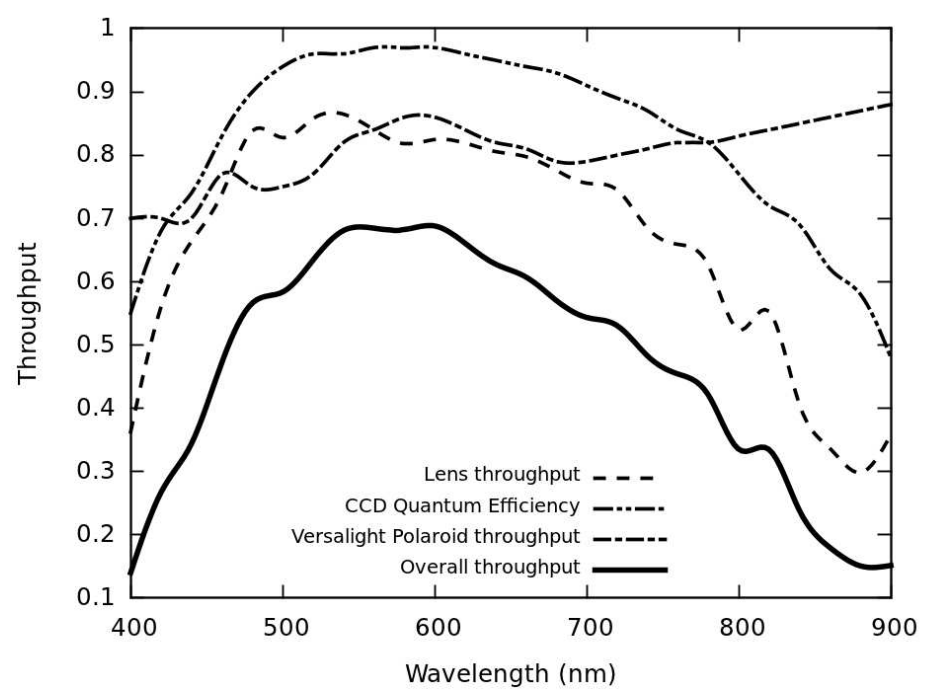

Figure 4. The throughput of the optical elements of RINGO3 linked with the CCD Quantum Efficiency (QE), provide an overall instrumental efficiency across the operating wavelengths. The Spectra of GRBs can then be put through this model to obtain the numbers of counts observable at each wavelength. Note: Shown here is just the BV type camera QE

region from the slit region. By doing this subtraction CCD bias and dark counts and any systematic background are accouted for. The baseline data was reduced by counting the full flux in each frame. A dark frame (with monochromator off) was also taken and the counts subtracted from the baseline frame and subtracted off. Due to the different optical set-ups of slit and baseline observations, as well as the differing illuminations of the $24 \mathrm{~V}$ bulb and 5V LED, the observations were not directly comparable. Instead an arbitrary scaled throughput was 
calculated by dividing each wavelength value of flux with the lenses by the baseline fluxes. This division removes the effects of quantum efficiency of the CCD and monochromator output spectra, just leaving a throughput profile of the lenses. These curves were then normalised and joined by making the reasonable assumption that each lens had a $92 \%$ throughput at $550 \mathrm{~nm}$, the centre of the visible spectrum, giving a maximum throughput of $85 \%$ with the two lenses at $550 \mathrm{~nm}$.

\subsection{Instrumental Efficiency and GRB Spectra Modelling}

With the measured throughput of the lenses, data for the EMCCD camera QE and throughput of the Versalight polaroid we created the overall instrumental efficiency as shown in Figure 4. Observations of early-time (first 15 minutes) GRB optical spectra are non-existent. However, we know that synchrotron emission is responsible for the majority of emission, so the spectrum will trace a power-law which represents the kinetic energy distribution of the electrons in the jet plasma. Hence we looked for suitable early time photometric measurments in BVRI bands, and a spectrum could be deduced from a best line fit of the fluxes of these measurements. This was performed on the best dataset we could find from the LTs observation of GRB $050502^{9}$ with BVRI measurements all taken within 15 minutes of the burst trigger. The non-simultaneous nature of these observations over a time when the GRB afterglow is fading yield an inaccurate result of only one object. We went back to the theoretical spectra of GRBs from the fireball model, ${ }^{10}$ which provides a power law optical spectrum for fast cooling (which occurs at early times) of Flux $\propto \nu^{-1 / 2}$ and therefore Counts $\propto \lambda^{1.5}$ when converted into wavelength. As shown in Figure 5 the theoretical spectra for fast and slow cooling confirm our photometric best fit as being reasonable. It is worth noting the model GRB spectra do not account for any absorption or reddening due to either the local circumburst medium or our own galactic ISM.

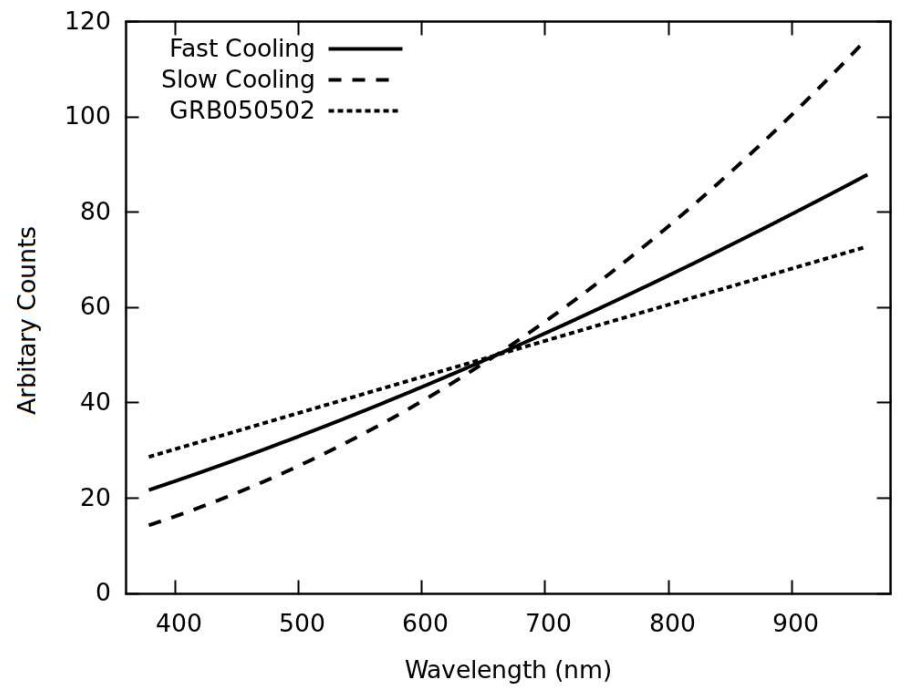

Figure 5. Models of GRB Spectra used in our waveband analysis. The Fast and Slow cooling curves are from Sari, Piran and Nayaran, ${ }^{10}$ and GRB050502 curve is a best fit from photometric measurements in BVRI bands on the LT from Guidorzi et al 2005. ${ }^{9}$ All curves are normalised at $660 \mathrm{~nm}$ for comparison and are scaled to photon counts after taking into account varying photon energies.

\subsection{Calculated Wavebands}

Our spectra for both the GRBs and the La Palma sky were converted into counts per wavelength bin and this was put through our instrumental efficiency model. The differing wavebands (and hence dichroic) cut-offs were definied by ensuring each band had $\sim 33 \%$ of the counts. This model is slightly flawed as we will get GRB and sky emission creating counts outside of the wavelength range modelled (400-900nm). However, this will only be a small contribution due to the rapidly falling instrumental efficiencies outside of this range. 
The result of this analysis is shown in Table 1. Initial ideas were for RINGO3 to have close to standard BVR bands, however mainly due to the characteristic colour of GRB afterglows we end up with 3 bands best described as a BV, R and I. The close agreement of the differing GRB models show that the instrumental efficiency has a large effect on the overall waveband selection. The counts from the sky are also in agreement with wavelength bands, ensuring that we will have similar sky noise in each band too. Our choice of using the BB camera has overall had little effect on the efficiency of the instrument, as any gain in efficiency achieved at the blue end is lost as after $\sim 500 \mathrm{~nm}$ it's efficiency is less than the BV camera (Figure 2). We are currently ordering the production of the dichroic mirrors, and are deciding the exact cut-off wavelengths in consultation with the suppliers.

Table 1. Calculated wavebands which give equal detected counts for GRB spectra and our dominant sky noise.

\begin{tabular}{|l|c|c|c|}
\hline Input Spectra & BV Band $(\mathrm{nm})$ & R Band (nm) & I Band (nm) \\
\hline GRB 050502 & $400-680$ & $680-770$ & $770-900$ \\
\hline Fast Cooling & $400-640$ & $640-740$ & $740-900$ \\
\hline Slow Cooling & $400-660$ & $660-760$ & $760-900$ \\
\hline La Palma Sky & $400-620$ & $620-770$ & $770-900$ \\
\hline
\end{tabular}

\section{CONCLUSIONS}

Currently RINGO3 is scheduled to be comissioned on the Liverpool Telescope in September 2012, where the optics will be characterised using polarmetric standard stars. ${ }^{11}$ Our good knowledge of the instrumental characteristics of RINGO2 will allow us to determine the polarimetric footprint of the dichroic mirrors. The three bands will need to be characterised also, so that photometry performed with RINGO3 can be convereted to more common photometric standards.

One area of investigation that we are keen to take up with RINGO3, is to measure the effect of different lunar phases and positions with relation to polarimetry. The reflected radiation from the moon will have a polarisation signature and hence we expect any sky background contribution from the moon to be polarised. This is especially apt for transient sources which can occur at any lunar phase.

\section{ACKNOWLEDGMENTS}

We acknowledge funding for the RINGO3 Project from the UK Science and Technology Facilities Council.

\section{REFERENCES}

[1] Steele, I. A., Smith, R. J., Rees, P., Baker, I. P., Bates, S. D., Bode, M. F., Bowman, M. K., Carter, D., Etherton, J., Ford, M. J., Fraser, S. N., Gamboc, A., Lett, R. D. J., Mansfield, A. G., Marchant, J. M., Medrano-cerda, G. A., Mottram, C. J., Raback, D., Scott, A. B., Tomlinson, M. D., and Zamanov, R., "The liverpool telescope: performance and first results," in [Ground-based and Airborne Instrumentation for Astronomy], Oschmann, J. M., ed., Proc. SPIE 5489, 679-692 (2004).

[2] Guidorzi, C., Monfardini, A., Gamboc, A., Mundell, C. G., Steele, I. A., Carter, D., Bode, M. F., Smith, R. J., n. Fraser, S., Burgdorf, M. J., and m. Newsam, A., "The automatic real-time gamma-ray burst pipeline of the $2 \mathrm{~m}$ liverpool telescope," PASP 118, 288-296 (2006).

[3] Steele, I. A., Bates, S. D., Guidorzi, C., Mottram, C. J., Mundell, C. G., and Smith, R. J., "Ringo: a nocel ring polarimeter for rapid grb followup," in [Ground-based and Airborne Instrumentation for Astronomy], Moorwood, A. F. M.; Iye, M., ed., Proc. SPIE 5492, 677-688 (2004).

[4] Steele, I. A., Bates, S. D., Guidorzi, C., Mottram, C. J., Mundell, C. G., and Smith, R. J., "Ringo2: an emccd-based polarimeter for grb followup," in [Ground-based and Airborne Instrumentation for Astronomy III], McLean, Ian S.; Ramsay, S. K. T. H., ed., Proc. SPIE 7735, 773549 (2010).

[5] Clarke, D. and Neumayer, D., "Experiments with a novel ccd stellar polarimeter," $A \mathscr{G} A$ 383, 360-366 (2002). 
[6] Bessell, M. S., "Ubvri passbands," PASP 102, 1181-1199 (1990).

[7] Greiner, J., Bornemann, W., Clemens, C., Deuter, M., Hasinger, G., Honsberg, M., Huber, H., Huber, S., Krauss, M., Krühler, T., Küpcü Yoldaş, A., Mayer-Hasselwander, H., Mican, B., Primak, N., Schrey, F., Steiner, I., Szokoly, G., Thöne, C. C., Yoldaş, A., Klose, S., Laux, U., and Winkler, J., "GROND: a 7-Channel Imager," PASP 120, 405-424 (2008).

[8] Benn, C. R. and Ellison, S. L., "Brightness of the night sky over la palma," New Astronomny Reviews 42, 503-507 (1998).

[9] Guidorzi, C., Monfardini, A., Gamboc, A., Mundell, C. G., Steele, I. A., Carter, D., Bode, M. F., Smith, R. J., Mottram, C. J., Burgdorf, M. J., Tanvir, N. R., Masetti, N., and Pian, E., "The early multicolor afterglow of grb 050502a: Possible evidence for a uniform medium with density clumps," ApJ 630, L121L124 (2005).

[10] Sari, R., Piran, T., and Narayan, R., "Spectra and lightcurves of gamma-ray burst afterglows," ApJ 497, L17-L20 (1998).

[11] Schmidt, G. D., Elston, R., and Lupie, O. L., "The hubble space telescope northern-hemisphere grid of stellar polarimetric standards," AJ 104, 1563-1567 (1992). 